Methods Using data from the Provincial Laboratory and STI clinic, an anonymized dataset with the last HIVAb- (HIVGO1/2, Abbott, AxSym +/- Western Blot) (STI clinic patients) or first syphAb+ (Architect, Abbott +/- RPR \& Innolia) was constructed with: (1) All patients: age, gender, date of testing, N. gonorrhoea (NG) and C. trachomatis co-infection within 30 days of HIV/syphilis test, infectious syphilis stage, and HIV testing as of Dec 2010 and (2) STI clinic patients only: syphilis test results within 30 days of their HIVAb- test. Patients remaining HIVAb-> 180 days after the sample receipt date were excluded from HIV pNAT. The remaining samples were divided into SyphAb+ and SyphAb-subsets. Pools of 25 samples were tested using the Roche COBAS AmpliPrep/COBAS TaqMan HIV-1 Test (pNAT). Positive pools were broken down to identify positive individuals. Percentage calculations were based on patients with pNAT.

Results 7954 HIVAb- patients were eligible. Of these, 2237 were retested and were HIVAb- > 180 days; 216 (10\%) of this subset were SyphAb+. 5441 (95\%) of the remaining patients had samples available for pNAT: 5001 were SyphAb-, 331 were SyphAb+, and 109 had no syphilis testing. Four SyphAb+ patients $(0.07 \%$ of all, $1.2 \%$ of SyphAb+), all seen at STI clinic, had detectable HIV RNA using pNAT; one patient had Early Latent Syphilis and positive NG culture.

Conclusions pNAT testing can be used to identify acute HIV infections in high risk populations. Patients with positive syphilis serology may be an important subset for this approach.

\section{P3.251 SYPHILIS AND HIV CO-INFECTION IN PATIENTS ATENDING AN AIDS OUTPATIENT CLINIC IN VITORIA, BRAZIL}

doi:10.1136/sextrans-2013-051184.0707

${ }^{1}$ A E Miranda, 'F M Callegari, 'L F Pinto Neto, ${ }^{2} \mathrm{~K}$ Page. 'Universidade Federal do Espirito Santo, Vitoria, Brazil; 'University of california San Francisco, San Francisco, CA, United States

Background HIV-positive patients should be regularly screened for syphilis. Detection and treatment of syphilis can help to reduce HIV transmission.

Objective Our goal was to determine the prevalence of and associated factors with syphilis in HIV patients attending an AIDS outpatient clinic in Vitoria, Brazil.

Methods Cross-sectional study was conducted in HIV/AIDS patients. They were interviewed for demographic, behavioural and clinical characteristics and had blood collected (venipuncture and fingertick) for VDRL and treponemal tests (rapid test) after signing an informed consent.

Results A total of 438 patients were included in the study. Over half $(55 \%)$ of the sample was male, mean age was 43 years ( $\mathrm{SD} \pm 11$ years), and mean years of schooling was 8.1 ( $\mathrm{SD} \pm 4.2$ years). The prevalence of syphilis was $5.3 \%$ (CI 95\% 3.3\%-7.3\%). The treponemal test was positive in $18.9 \%$ of participants. Over one third $(37.8 \%)$ of the male patients reported a history of sex with men (MSM). No women reported homosexual behaviour. More than two-thirds (70\%) of the participants reported consistent condom use in the last year; $6.4 \%$ were sexworkers and $25.8 \%$ were illicit drug users. A total of $72.4 \%$ reported having had one sexual partner in the last year In multivariate analysis, syphilis was associated with male gender [OR $=4.57(\mathrm{CI} 95 \%: 1.03-20.0])$, men who have sex with men $[\mathrm{OR}=1.78(\mathrm{IC} 95 \%: 1.64-4.14)]$, not on antiretroviral therapy $[O R=0.18$ (CI 95\% 0.06-0.59], and history of previously treated syphilis infection [OR 5.54 (CI95\%:1.95-15.76].

Conclusions Our finding highlights the importance of preventing and promptly treating syphilis in people living with HIV/AIDS. Patients with HIV/AIDS must be screened and monitored annually for early detection of syphilis, to provide early treatment and follow-up to avoid reinfections.

\section{P3.252 STI SURVEILLANCE WITHIN THE GENERAL POPULATION AND IN AIDS REFERENCE CENTRES (ARC) IN BELGIUM: CIRCUMSTANCES OF HIV TESTING IN PATIENTS DIAGNOSED WITH AN STI}

doi:10.1136/sextrans-2013-051184.0708

R Verbrugge, J Deblonde, D Van Beckhoven, A Sasse. Scientitfic Institute for Public Health, Brussels, Belgium

Background Sentinel STI surveillance with clinicians exists since 2000. STI within HIV patients triggered STI surveillance in ARC, specialised in care of HIV patients and patients at risk. No STI-HIV screening guidelines exist. STI is a driving force for HIV epidemics. Methods STI surveillance registers STI episodes, including questions on HIV serology, HIV testing and risk behaviour. HIV testing circumstances are analysed in both networks.

Results In 2011, 744 STI patients were registered by the network of clinicians and 250 STI coinfected HIV patients by the ARC.

HIV testing circumstances are known for $95 \%$ of the STI patients from the clinicians network. $51 \%$ was tested during STI consultation, 19\% were never tested, being postponed, refused or not proposed. The remaining 30\% was tested previously of whom $49 \%$ was HIV negative and not retested.

Within the clinicians network, HIV serology was reported in $79 \%$ of the cases: $21 \%$ are HIV positive of whom $14 \%$ discovered their seropositivity at STI consultation.

Within the ARC, 7\% of the coinfected HIV patients discovered their HIV positivity simultaneously with the STI diagnosis. Among STI patients in the ARC with at first negative/unknown HIV status, $57 \%$ was tested for STI-HIV simultaneously: $15 \%$ were found HIV positive. $11 \%$ of the seronegative STI patients were not retested. For the other $32 \%$ of the STI patients, their HIV status remains unknown.

Conclusions Surveillance of STI in both networks shows that HIV testing is not always performed during STI consultation, the HIV status may remain unknown and/or patients are not retested. Nevertheless, HIV positivity and STI are regularly found simultaneously.

Knowing the HIV status of STI patients and screening HIV patients for STI is important for reducing HIV-STI transmission. National STI-HIV screening guidelines should be developed and implemented.

Besides screening, condom use remains the most important preventive factor

P3.253 SERO PREVALENCE OF HIV, HEPATITIS B, HEPATITIS C AND SYPHILIS AMONG BLOOD DONORS IN KATHMANDU, NEPAL

doi:10.1136/sextrans-2013-051184.0709

D R Pandeya. Nepalese Army Institute of Health Sciences, Kathmandu, Nepal

Background Mandatory screening tests are performed for human HIV1/2, Hepatitis B and C and Syphilis by blood transfusion centres in Nepal because blood transfusion is the most efficient mode of transmission of these diseases. The study is aimed to determine the sero-prevalence of these four Transfusion transmissible infections. Methodology A cross-sectional retrospective study was conducted at Tribhuvan University teaching Hospital, Maharajgunj during the period from July 1st 2007 to June 30th 2011. Total of 11160 samples from Volunteer and replacement donors were screened for Anti-HIV, Anti-HCV and HBsAg by ELISA methods. The Reactive cases were confirmed by confirmatory method as per the national algorithm.

Results The prevalence of HIV, HBV, HCV and syphilis were determined to be $0.17 \%, 0.44 \%, 0.71 \%$ and $0.35 \%$ respectively. HBV and HCV sero-prevalence was found to be higher among male blood donors but, Syphilis and HIV prevalence was higher in female 